\title{
Simulation of Ultra-slow Oscillations Using the Integrate and Fire Neuron Model
}

\author{
Danny $\mathrm{Ng}^{1}$, MokSiew-Ying ${ }^{1}$, Chan Siow-Cheng ${ }^{1}$, Goh Sing-Yau ${ }^{2}$ \\ ${ }^{1}$ Department of Mechatronics and BioMedical Engineering, University Tunku Abdul Rahman, UTAR, Kuala Lumpur, Malaysia \\ ${ }^{2}$ Department of Mechanical and Material Engineering, University Tunku Abdul Rahman, UTAR, Kuala Lumpur, Malaysia \\ Email: ngwk@utar.edu.my, moksy@utar.edu.my, chansc@utar.edu.my, gohsy@utar.edu.my
}

Received 2012

\begin{abstract}
The Integrate and Fire (IF) neuron model wasusedto simulate ultra-slow oscillations that were observed in cortical cultures. Simulation of a network with 2 sub-networks is conducted in this study. We introduced an additional equation that governs the generation and dissipation of an inhibitory property to each of the sub-network.Sub-networks that fire at different rate are generated from the simulation. The network activity from the simulation oscillates at frequencies that are comparable to ultra-slow oscillations observed in cortical cultures.
\end{abstract}

Keywords: Integrate and Fire Neurons; Ultra-slow Oscillation; Simulation

\section{Introduction}

Oscillations play a crucial role in numerous processes of the nervous system. Oscillations in the form of electroencephalogram (EEG) are present in different brain structures, with frequencies ranging from $0.5 \mathrm{~Hz}$ ( $\delta$ rhythm) to $40-80 \mathrm{~Hz}$ ( $\gamma$ rhythm), and even up to $200 \mathrm{~Hz}$ [1]. Slow oscillations with frequency less than $1 \mathrm{~Hz}$ were detected in various in vivo experiments [2-4]. Ultra-slow oscillations with frequencies less than $0.01 \mathrm{~Hz}$ were reported in other experiments [5-8]. A recent study by Mok et al. [5] reported on ultra-slow oscillations in MEA cultures of rat cortical neurons. The ultra-slow oscillations were characterized by large synchronized bursts at the peaks and smaller bursts at the troughs. These activity patterns emerged in cultures after the fourth week in vitro.

In computational studies, it is possible to generate global oscillations in a network of inhibitory neurons [9]. Inhibitory coupling in the network can act to synchronize the oscillatory activity in the network [10]. Heterogeneous networks consisting of inhibitory and excitatory neurons can exhibit a wide range of behavior depending on the parameters and inputs given to the network [11,12]. Activity patterns such as steady firing and bursting can be simulated by varying the network connectivity and fractions of endogenously active neurons $[13,14]$. Bursting activity can be observed by having a balance between the excitation and inhibition in the network [15]. Synaptic characteristics such as connection strength and synaptic depression between neurons in the model can influence the burst activity pattern of a network [16]. For some large networks, the synchronized bursting events might be classified into several distinct types based on their spatiotemporal substructures [17].

\section{Simulation}

In this study, numerical simulations of a network of IF neurons

* This workwas supported by a grant from the Fundamental Research Grant Schemeunder the Ministry of Higher Education Malaysia. were conducted in an attempt to simulateultra-slow oscillations observed by Mok et al. [5]. All equations in this simulation were solved using the Runge-Kutta 4th order method [18].

\subsection{Neuron Model}

The single neuron model introduced by Lathamet al. [13] was used in this simulation. The time evolution equation for the membrane potential of neuron $v_{i}(t)$ is,

$$
T_{m} \frac{d v_{i}(t)}{d t}=\alpha\left(v_{i}(t)-v_{r}\right)\left(v_{i}(t)-v_{t}\right)+I_{a, i}(t)-I_{A H P}-I_{S Y N}
$$

where $T_{m}$ is the membrane time constant, $\alpha$ determines the rate of change for the membrane, $v_{r}$ is the resting potential, $v_{t}$ is the threshold potential, $I_{a, i}(t)$ controls the fraction of endogenously active cells, $I_{A H P}$ the afterhyperpolarization (AHP) current and $I_{S Y N}$ the synaptic current.

The AHP current is a combination of the fast AHP current which is responsible for the refractory period and slow AHP current which is responsible for spike frequency adaptation. $I_{A H P}$ is given as,

$$
I_{A H P}=\left(g_{k, i}+g_{k-c a, i}\right)\left(v_{i}(t)-\varepsilon_{k}\right)
$$

where $\varepsilon_{k}$ is the potassium reversal potential. $g_{k, i}$ and $g_{k-c a, i}$, the potassium conductance is governed by the time evolution equation,

$$
\begin{gathered}
\frac{d g_{k, i}}{d t}=-\frac{g_{k, i}}{\tau_{K, i}}+\delta_{k, i} \sum_{\mu} \delta\left(t-t_{i}^{u}\right) \\
\frac{d g_{k-C a, i}}{d t}=-\frac{g_{k-C a, i}}{\tau_{k-C a, i}}+\delta_{k-C a, i} \sum_{\mu} \delta\left(t-t_{i}^{u}\right)
\end{gathered}
$$

where $\tau_{K, i}$ and $\tau_{k-C a, i}$ are the time constant, $\delta_{k, i}$ and $\delta_{k-C a, i}$ are the increase in conductance and $t_{i}^{u}$ is the time spike occur on neuron $i$. The synaptic current $I_{S Y N}$ is given as 


$$
I_{S Y N}=\left(v_{i}(t) \tilde{I}_{i}-\tilde{I}_{\varepsilon, i}\right)
$$

where $v_{i}(t)$ is the membrane potential. $\tilde{I}_{i}$ and $\tilde{I}_{\varepsilon, i}$ are given as

$$
\begin{gathered}
\frac{d \tilde{I}_{i}}{d t}=-\frac{\tilde{I}_{i}}{\tau_{s}}+r_{s} \sum_{j, u} w_{i j} \delta\left(t-t_{j}^{u}\right) \\
\frac{d \tilde{I}_{\varepsilon, i}}{d t}=-\frac{\tilde{I}_{\varepsilon, i}}{\tau_{s}}+r_{s} \sum_{j, u} w_{i j} \varepsilon_{j} \delta\left(t-t_{j}^{u}\right)
\end{gathered}
$$

where $\tau_{s}$ is the decay constant for number of open channel, $r_{s}$ is the number of closed channel open during spike, $w_{i j}$ the strength of connection and $\varepsilon_{j}$ the reversal potential.

\subsection{Network Model}

Network connections are formed randomly between neurons. Connectivity bias [13] are introduced in the network through

$$
\begin{gathered}
P_{E T_{j}}=\frac{K_{T_{j}}}{N_{E}+N_{I} B_{T_{j}}} \\
P_{I T_{j}}=\frac{K_{T_{j}} B_{T_{j}}}{N_{E}+N_{I} B_{T_{j}}}
\end{gathered}
$$

where $P_{E T_{j}}$ and $P_{I T_{j}}$ are the connection probability for excitatory and inhibitory neurons, $K_{T_{j}}$ is the number of postsynaptic neuron, $N_{I}$ is the number of inhibitory neurons, $N_{E}$ is the number of excitatory neurons, $B_{T_{j}}$ is the connection bias and $T_{j}$ is the type of postsynaptic neuron. The connection probability with inhibitory neuron will be higher if $B_{T_{j}}>$ 1 whereas connection probability with excitatory neuron will be higher if $B_{T_{j}}<1$. Neurons in the network are allowed to connect to all other neurons.

\subsection{Sub-networks}

Single-unit activity from the experiment showed that some neurons fire continuously while others fire only at the peaks. Similar burst motifs were observed in experiment conducted by Mok et al. [5] and Volman et al. [17]. Based on the activity pattern of the single-unit activity and the burst motifs [5], we postulate that there are two or more distinct sub-networks in the cortical culture.

For the purpose of the present simulations, the neurons are divided into 2 sub-networks.Neurons are assigned randomly to the sub-networks through the Bernoulli processwith

$$
p=\frac{N_{s 1}}{N}
$$

where $p$ is the probability of success, $N_{s 1}$ is the number of neurons in sub-network $1, N$ is the total number of neurons. A success in the trial will place the neuron in sub-network 1 whereas a fail in the trial will place the neuron in sub-network 2 .

An equation describing the generation and dissipation of an inhibition property,

$$
\frac{d \varphi}{d t}=-\frac{\varphi}{\tau_{\varphi}}+\delta_{\varphi} \sum_{\mu, i=0}^{i=n} \delta\left(t-t_{i}^{u}\right)
$$

is assigned to each of the sub-network. $\varphi$ increaseswhen neuron spikes in the sub-network and decays with a time constant, $\tau_{\varphi}$. The increase in inhibition property is given by $\delta_{\varphi}$. When $\varphi$ reaches anupper threshold $\left(T_{u}\right)$, the neurons within the sub-network stop firing. The neurons start to fire again when the inhibitory property has dissipated to alower threshold $\left(T_{l}\right)$. In this manner we will have neurons in each sub-network firing at a different rate.

\section{Results and Discussion}

The parameters in Table 1 are used in the simulation to reproduce the bursting activity seen in the experiments. Figure 1 show simulated activities with frequencies similar to those observed in the experiments. For the simulation, a slow $\tau_{\varphi}$ is assigned to sub-network 1 to simulate a neuron group that active only at the peaks whereas a fast $\tau_{\varphi}$ is assigned to subnetwork 2 to simulate a neuron group that is active all the time. Figure 2 shows simulated activities compared to another set of experiment. With a different number of neuron in the sub-networks, peak activity width of around 100s can be obtained from the simulation. Upper threshold, $T_{u}=3.8$ andlower threshold, $T_{l}=0.1$ are used for the simulations.

If the properties of the 2 sub-networks are different, it is possible to simulate one sub-network that fires continuously while another sub-network fires periodically. The time period of

\begin{tabular}{|c|c|}
\hline Parameter & Value \\
\hline$T_{m}$ & $10 \mathrm{~ms}$ \\
\hline$v_{r}$ & $-65 \mathrm{mV}$ \\
\hline$v_{t}$ & $-50 \mathrm{mV}$ \\
\hline$\alpha$ & $1 / 15$ \\
\hline$\varepsilon_{k}$ & $-80 \mathrm{mV}$ \\
\hline$\tau_{\mathrm{s}}$ & $3 \mathrm{~ms}$ \\
\hline$r_{\mathrm{s}}$ & $0.1 \mathrm{mS}$ \\
\hline$\tau_{K}$ & $30 \mathrm{~ms}$ \\
\hline$\delta_{k}$ & $1 \mathrm{mS}$ \\
\hline$\delta_{k-C a}$ & $0.2 \mathrm{mS}$ \\
\hline Number of Neuron & 10000 \\
\hline Connection per Neuron & 2000 \\
\hline Excitatory Neuron & 2000 \\
\hline Inhibitory Neuron & 8000 \\
\hline $\mathrm{V}_{\mathrm{EPSP}}$ & $1 \mathrm{mV}$ \\
\hline $\mathrm{V}_{\text {IPSP }}$ & $-1.5 \mathrm{mV}$ \\
\hline $\mathrm{B}_{\mathrm{i}}$ & 0.8 \\
\hline $\mathrm{B}_{\mathrm{e}}$ & 1.2 \\
\hline $\mathrm{I}_{\max }$ & $3.8-5$ \\
\hline
\end{tabular}
the ultra-slow oscillations in experiments by Mok et al. [5] is much larger than previously reported and cannot be generated by the standard IF model. The current simulations of the ultra-slow oscillations are the results of 2 sub-networks firing at different rates.

Table 1. Parameters used for simulation of neuron activity. 


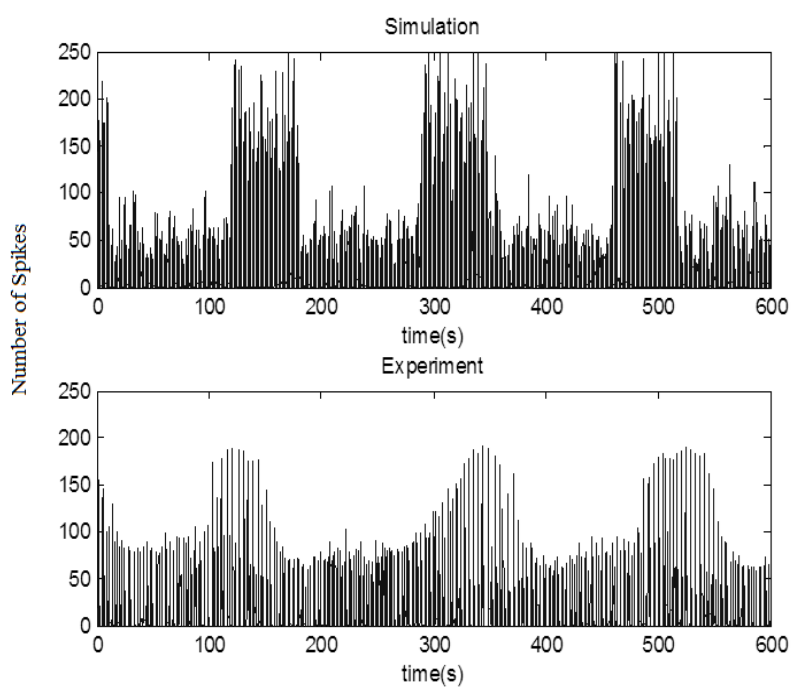

Figure 1. Simulation of activity generated with parameters: $\delta_{\varphi}=$ 0.07, upper threshold, $T_{u}=3.8$, lower threshold, $T_{1}=0.1$, subnetwork 1: number of neuron $=2000, \tau_{\varphi}=30000$, sub-network 2 : number of neurons $=8000, \tau_{\varphi}=1000$ compared with experimental results. The time bin for spikes is $10 \mathrm{~ms}$.

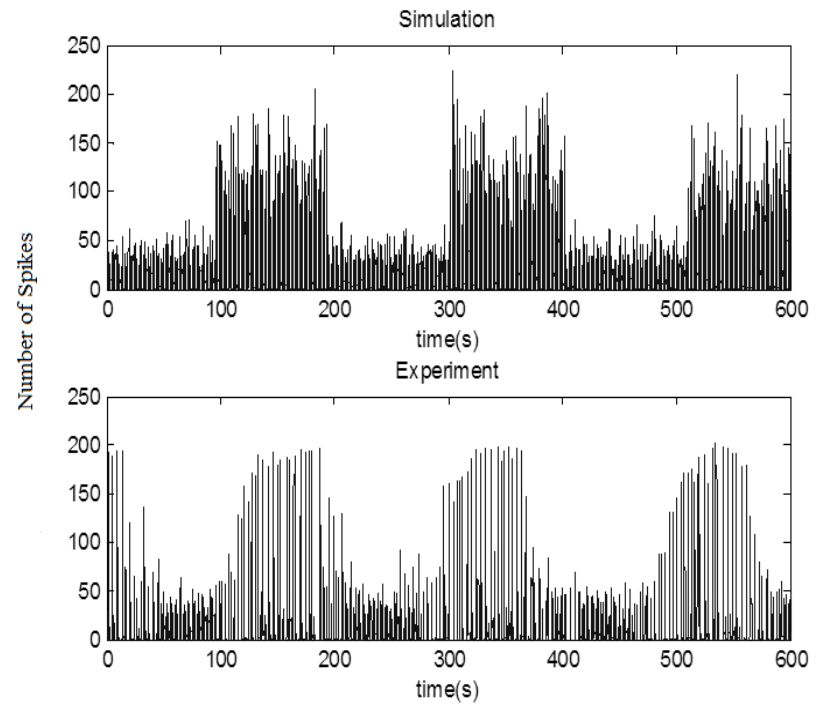

Figure 2. Simulation of activity generated with parameters: $\delta_{\varphi}=$ 0.07, upper threshold, $T_{u}=3.8$, lower threshold, $T_{1}=0.1$, subnetwork 1: number of neuron $=3000, \tau_{\varphi}=30000$, sub-network 2: number of neurons $=7000, \tau_{\varphi}=1000$ compared with experimental results. The time bin for spikes is $10 \mathrm{~ms}$.

It is likely that these ultra-slow oscillations are controlled by biochemical processes and/or network structures in the cortical culture. Further experiments are needed to better understand the underlying biochemical processes that cause theseultra-slow oscillations.

\section{REFERENCES}

[1] A. Husain, W.O. Tatum and P.W. Kaplan.Handbook of EEG interpretation.Demos Medical, 2008.

[2] M. Steriade, A. Nuñez and F. Amzica. "A novel slow ( $<1 \mathrm{hz})$ oscillation of neocortical neurons in vivo: depolarizing and hyperpolarizing components" J. Neuroscience, vol 13, issue 8 pp. 3252-3265, Aug 1993.

[3] F. Amzica and M. Steriade. "Short- and long-range neuronal synchronization of the slow $(<1 \mathrm{hz})$ cortical oscillation", $J$. of Neurophysiology, vol. 73, issue 1, pp. 20-38, Jan 1995.

[4] D. Contreras ,I. Timofeev and M.Steriade."Mechanisms of long-lasting hyperpolarizations underlying slowsleep oscillations in cat corticothalamicnetworks" J. of Physiology, vol. 494, pp 251-264, Jul 1996.

[5] S. Y. Mok, Z. Nadasdy, Y.M. Lim, S.Y. Goh. "Ultra-slow oscillations in cortical networks in vitro”, Neuroscience, vol. 206, pp 17-24, Mar 2012.

[6] M. Penttonen, N. Nurminen, R. Miettinen, J. Sirviö, D. A. Henze, J. Csicsvári, and G. Buzsáki. "Ultra-slow oscillation (0.025 hz) triggers hippocampal afterdischarges in wistar rats” Neuroscience, vol 94, issue 3, pp. 735-743, Oct 1999.

[7] S. Vanhatalo, J. M. Palva, M. D. Holmes, J. W. Miller JW, J. VoipioandK. Kaila. "Infraslow oscillations modulate excitability and interictal epileptic activity in the human cortex during sleep" Proc.of the National Academy of Sciences, vol. 101, pp. 5053-5057, Apr 2004

[8] P. J. Drew, J. H. Duyn,E.Golanov and D.Kleinfeld“Finding coherence in spontaneous oscillations"Nature Neuroscience vol. 11, pp 991-993, 2008.

[9] N. Brunel and V.Hakim. "Fast global oscillations in networks of integrate-and-fire neurons with low firing rates", Neural Computation, vol. 11, issue 7, pp. 1621-1671, Oct 1999.

[10] X. J. Wang and G. Buzsáki."Gamma oscillation by synaptic inhibition in a hippocampal interneuronal network model", $J$. Neuroscience, vol. 16, issue 20, pp. 6402-6413, Oct 1996.

[11] N. Brunel. "Dynamics of sparsely connected networks of excitatory and inhibitory spiking neurons", $J$ Computational Neuroscience, vol. 8, issue 3, pp. 183-208, Jun 2000.

[12] D. Hansel and G. Mato. "Asynchronous states and the emergence of synchrony in large networks of interacting excitatory and inhibitory neurons”, Neural Computation, vol. 15, issue 1, pp. 1-56, Jan 2003.

[13] P. E. Latham, B. J. Richmond, P. G. Nelson, and S. Nirenberg." Intrinsic dynamics in neuronal networks.I.Theory." J. of Neurophysiology, vol 83, issue 2, pp. 808-827, Feb 2000.

[14] P.E. Latham, B.J. Richmond, S. Nirenberg, and P.G. Nelson."Intrinsic dynamics in neuronal networks. II. Experiment” $J$. of Neurophysiology, vol. 83, issue 2, pp. 828-835, Feb 2000.

[15] P. Kudela, P.J. Franaszczuk, and G.K. Bergey.“Changing excitation and inhibition in simulated neural networks: effects on induced bursting behaviour", Biological Cybernetic, vol. 88, issue 4, pp. 276-285, Apr 2003.

[16] M. Tsodyks, A. Uziel, and H. Markram."Synchrony generation in recurrent networks with frequency-dependent synapses”, $J$. of Neuroscience, vol. 20, RC50, pp 1-5, Jan 2000.

[17] V. Volman, I. Baruchi, and E. Ben-Jacob."Manifestation of function-follow-form in cultured neuronal networks”, Physical Biology, vol.2, issue 2, pp. 98-110, Jun 2005.

[18] L.R Petzold and U.M. Ascher.Computer methods for ordinary differential equations and differential-algebraic equations.SIAM, 1998. 